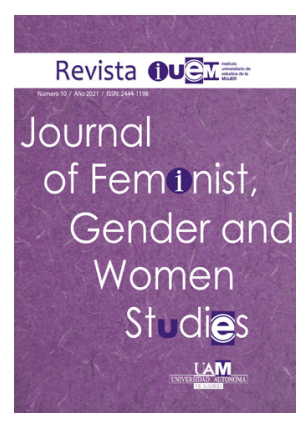

Recibido: $26 / 04 / 2020$

Aceptado: 19/02/2021

\section{«El embarazo es una máquina, no una mujer» Deshumanización y sexismo misógino en el planteamiento favorable al 'trabajo gestacional'}

\author{
"Pregnancy is a machine, not a woman» Dehumanization and misogynous sexism \\ in pro 'gestational work' approach
}

Lydia Delicado-Moratalla ${ }^{1, @ ~}$

${ }^{1}$ Departamento de Historia, Geografía y Arte. Universidad Jaume I.

@ Autor/a de correspondencia: Iydia.delicado@gmail.com

\begin{abstract}
Resumen
El 'embarazo profesional' y la 'gestante cyborg' podrían parecer parte de una distopía propia de un futuro transhumanista, en el que no se vislumbra la gestación de la vida humana sin la intervención tecnológica o sin su integración en las lógicas mercantilistas. Sin embargo, ya son una realidad en algunos discursos académicos del ámbito queer y trans. En este ensayo realizo una reflexión feminista política y abolicionista en torno a la gestación subrogada y a la subsiguiente idea de 'trabajo gestacional'. Poniendo en el centro al sujeto político mujer, parto de los argumentos de las pensadoras feministas críticas con esta práctica y me baso en las contribuciones etnográficas más destacadas sobre la temática. Analizo la deriva pro vientres de alquiler fijándome especialmente en los retos políticos que plantea con respecto a los derechos reproductivos de las mujeres. Examino el sexismo misógino que presenta neologismos como 'gestante profesional' y fórmulas similares. Compruebo que las propuestas favorables a la gestación subrogada tienen características clásicas de las prácticas androcéntricas, con una intención insistente por interponerse tecnológicamente en el proceso vital que es la gestación. Concluyo con la idea de que proletarizar y maquinizar la maternidad significa que se margina la experiencia humana para orientarse hacia el paradigma biocapitalista y ello conduce directamente a la deshumanización y a la explotación extrema de las mujeres.
\end{abstract}

Palabras clave: gestación subrogada, deshumanización, sexismo misógino, tecnofeminismo, mujeres.

\begin{abstract}
The 'professional pregnancy' and the 'cyborg gestator' could seem parts of a dystopian transhumanist future where the gestation of human life is not foreseen without neither the technological intervention nor its integration into the market logics. However, for a few academic discourses within the queer and trans arenas, these ideas are already a reality. In this essay I present an abolitionist and political feminist critique about gestational surrogacy and its subsequent idea of 'gestational work'. Focusing in woman as a political subject I draw on the ideas of critical feminist thinkers regarding this practice. I am also based on the most relevant ethnographic contributions about the issue. I analyse the pro wombs-for-rent flow by paying special attention to the political challenges that this position poses in relation to women's reproductive rights. I examine the misogynous sexism of the neologisms like 'professional gestator' and similar formulas. I prove that supportive proposals of gestational surrogacy contain classical androcentric characteristics as to the insistent intention of intervening technologically in the vital process that gestation is. I conclude with the idea that labouring and mechanizing maternity implies a marginalization of the human experience in order to orientate the process to the bio-capitalistic paradigm. Finally, this leads directly to dehumanization and to the extreme exploitation of women.
\end{abstract}

Keywords: gestational surrogacy, dehumanization, misogynous sexism, technofeminism, women. 


\section{INTRODUCCIÓN}

Una de las frases célebres del feminismo que cuenta con absoluta vigencia en la actualidad es aquella famosa de Angela Davis "el feminismo es la idea radical de que las mujeres son personas". Sophie Lewis, publica en 2017 su artículo Defending Intimacy Against What? Limits of Antisurrogacy Feminisms en la revista de pensamiento e investigación feminista Signs: Journal of Women in Culture and Society de la Universidad de Chicago, en el que otorga pleno apoyo a la práctica de los vientres de alquiler ${ }^{1}$. En su texto, elabora una argumentación para que la gestación subrogada sea conceptualizada como 'trabajo gestacional', al mismo tiempo que sostiene la fantasía de construir mujeres cyborg, la idea de disociar el embarazo de las mujeres y una apuesta por atribuir un carácter de fluidez de género a la gestación, especialmente a la comercial. Lewis (2017) se manifiesta absolutamente en contra del planteamiento abolicionista sobre los vientres de alquiler y del movimiento Stop Surrogacy Now -llegando a publicar su libro titulado Full Surrogacy Now (2019)-, para quienes uno de los lemas de mayor alcance ha sido "una mujer es un ser humano, no una máquina", expresión que está muy próxima a las palabras de Adrienne Rich (2019: 43) pues parece que resulta todavía necesario "afirmar el valor intrínseco humano de la mujer mientras este continúa siendo negado de forma insidiosa y flagrante".

Como examinaré en este ensayo, los discursos favorables a la tecnología reproductiva suelen contener una idea de que la gestación sin intervención tecnológica es una fantasía nostálgica y errática, incluso normativa y transfóbica (Lewis, 2017). Aesta idea se le han sumado otras, conformando un apoyo teórico al desarrollo del biocapitalismo tecnificado. Desde algunos sectores académicos se mantiene una línea discursiva que defiende no sólo la gestación artificial, sino la atribución de 'trabajo gestacional' al embarazo que lleva a cabo una mujer en un contexto de gestación subrogada. Para desarrollar ese marco interpretativo, Sophie Lewis (2017), disemina un lenguaje que evita al máximo el uso de la palabra mujer unida al embarazo. Esta línea de pensamiento ya ha causado influencia en la opinión pública. La expresión 'gestante' en sustitución de 'mujer que gesta' aparece recogida, críticamente, en el libro "Gestación subrogada. Capitalismo, patriarcado y poder" de Layla Martínez (2019) y podemos ver que en reportajes televisivos, como Nens que no venen de París (CCMA, 2016) se habla de 'gestante' en vez de mujer embarazada. Como explica Rosa María Rodríguez (2019: 132), en la deriva trans que apoya los vientres de alquiler "se evitará hablar de embarazo o parto e incluso de mujer, que pasa a ser "persona gestante»". Me preocupa la alianza que se establece entre el discurso discriminatorio de Lewis (2017) contra las mujeres y la discriminación de las mujeres como forma fundamental de dominación en las sociedades patriarcales. Si no nombramos que la gestación subrogada es una forma de explotación específica de las mujeres, no realizamos un análisis riguroso. Y si existe una intención explícita de esconder dicha explotación con el eufemismo 'gestante' o 'trabajadora gestacional', se comete

1 Se emplearán las denominaciones 'vientre de alquiler', 'gestación subrogada', 'gestación para otros' y 'gestación comercial' indistintamente. una infravaloración de la compleja problemática política que hay detrás de esta estructura de servidumbre femenina que es la industria de los vientres de alquiler. Así, puesto que el lenguaje es una forma de determinar cuál es el papel de las mujeres en las realidades del mundo, las denominaciones que eliminan intencionadamente la participación de éstas en la práctica de los embarazos comerciales arrojan nuevos retos políticos y culturales que debemos analizar. Al mismo tiempo, encuentro que este esfuerzo extenuante por despreciar el papel imprescindible de las mujeres en el embarazo obedece a un sexismo misógino, concepto que desarrollaré en este texto.

Al tiempo que se suceden los debates académicos y sociales, el negocio de la gestación subrogada es floreciente. Ya en 2008 los cálculos apuntaban unas ganancias de seis billones de dólares anuales en todo el mundo (Oksala, 2019 citando a Smerdon, 2008). Tanto el feminismo académico como el activista han prestado atención a estos desarrollos desde los inicios de las técnicas de reproducción asistida a finales de los setenta del pasado siglo. En especial, las estudiosas feministas de Feminist International Network of Resistance to Reproductive and Genetic Engineering (FINRRAGE por sus siglas en inglés), una red internacional de activistas con preocupación por los impactos de las tecnologías reproductivas en las mujeres, describieron una particular inquietud por el impacto de estas técnicas y prácticas sobre los cuerpos y las vidas de las mujeres y sobre los aun no logrados (ni entonces ni ahora), derechos reproductivos plenos.

Una de las primeras obras que fueron radicalmente críticas con las tecnologías reproductivas fue "The Mother Machine", escrita por Gena Corea (1988), en la que defendió la idea de que estas tecnologías, así como la consideración y uso de las mujeres como máquinas de gestación, tenían un marcado significado político. Entre sus conclusiones, contemplaba que las tecnologías reproductivas aparecían como una creación que servía a los intereses del patriarcado y que convertía a las mujeres en objetos. Por su parte, FINRRAGE pensaban que la subrogación de la gestación reducía a las mujeres a meras vasijas, conformándose como un asalto a su dignidad, una forma de esclavitud y un riesgo para su salud.

Laura Nuño (2016) detecta que los cuerpos de las mujeres y sus capacidades reproductivas son puestas al servicio de los mercados globales, bajo un modo de producción reproductiva, en el que la reproducción se organiza en una cadena tecno-industrial, iniciada en un laboratorio, posteriormente deslocalizada a un útero del Sur Global y cuyo producto resultante, un ser humano, es adquirido por una familia o individuo del Norte Global. Alicia Puleo (2017: 181), entiende que el alquiler de mujeres gestantes se ha constituido como una práctica que "permite profundizar la colonización de los cuerpos en la búsqueda insaciable del beneficio económico", surgida en el seno de la desigualdad y del extractivismo, un mercado en el que las mujeres entran como seres profundamente sexuados, por ser mujeres en la plena conceptualización patriarcal.

La reflexión que expongo en este artículo se enmarca teóricamente dentro de la crítica feminista a determinados planteamientos y usos de la tecnología reproductiva, así como a los discursos académicos alineados en sus lógicas. 
Examino las formas en las que la gestación subrogada y la plática, derivada discriminan y deshumanizan a las mujeres. En las secciones siguientes, presento los argumentos de las pensadoras feministas críticas con la gestación subrogada y resumo algunas de las contribuciones etnográficas más destacadas sobre la temática. También, reflexiono críticamente sobre el marco de interpretación del 'trabajo gestacional' y estudio el significado del sexismo misógino intrínseco a la invención de los neologismos diseminados por Lewis (2017).

\section{LAS CRÍTICAS FEMINISTAS HACIA LA GESTACIÓN SUBROGADA}

Un punto de partida acertado para examinar los impactos de la gestación subrogada en la situación de las mujeres es comenzar por el análisis feminista de la tecnología reproductiva. Las feministas radicales entendieron en la década de los ochenta que las técnicas que se habían diseñado para la reproducción asistida invadían y abusaban los cuerpos de las mujeres. Constataban que dichas tecnologías estaban extendidamente controladas por un manejo androcéntrico de los procesos de fertilidad, en los que los cuerpos de las mujeres se convertían en espacios de explotación para el beneficio de un tejido clínico basado en el negocio, en cuyo mando estaban hombres poderosos tomando decisiones de calado sobre lo que sucedía en los cuerpos femeninos. Janice Raymond (1989) explica que la posición que adoptaron las feministas radicales frente a las tecnologías reproductivas ponía en el centro del debate la importancia de que fuesen las mujeres quienes pudieran tener el control sobre sus cuerpos. Insistían en que el ejercicio de la soberanía del propio cuerpo no podía significar otorgar el control a otros. Exigían el avance en los derechos reproductivos y el progreso de la investigación en prácticas de salud reproductiva que no fuesen dañinas ni marcadas por los intereses de la industria farmacéutica.

En su libro "Tecnofeminismo", Judy Wajcman (2006) apela a la reconsideración radical de cómo los procesos tecnológicos impactan en la cultura y se inquieta al observar los cambios en el significado de ser humano que han ido llegando con las tecnologías biomédicas. Para esta autora, las tecnologías reproductivas son pensadas en el seno de un modelo institucionalizado de poder y autoridad y considera conveniente estudiar la tecnología desde sus cualidades políticas. El análisis feminista de la tecnología explora aquellos abusos que se realizan con perjuicio hacia las mujeres, siendo las tecnologías reproductivas una experiencia concreta para éstas, en un contexto en el que la vida se está rediseñando en los laboratorios científicos.

Esta es la línea de pensamiento que defiende Maria Mies, para la que ni la ciencia ni la tecnología son neutrales desde un punto de vista de género. En sus escritos publicados en el recopilatorio "Ecofeminismo" (2014), editado por primera vez en 1993, se concentra fundamentalmente en la elaboración de una crítica a la tecnología reproductiva y a la gestación subrogada. Explica que esta tecnología ha intercedido en el proceso creativo humano espontáneo que es la fecundación, la gestación y el parto, para convertirlo en un acto artificial, enormemente medicalizado, que no tiene en cuenta los impactos sobre la salud de las mujeres. Desde sus tesis cree que el punto de despegue de las tecnologías reproductivas es la necesidad de expansión hacia nuevos formatos de mercado capitalista. De manera que los cuerpos femeninos aparecen en este contexto como un espacio de inversión, en el que poder experimentar y generar beneficios, pero no atiende a una necesidad terapéutica, "sino al hecho de que el capital y la ciencia necesitan a las mujeres para seguir manteniendo su modelo de crecimiento y de progreso" (Mies y Shiva, 2014: 290). El desarrollo de estas técnicas tampoco llega en una situación de igualdad para hombres y mujeres, sino en un escenario en el que las relaciones entre unos y otras están estructuradas en la dominación, la explotación y la subordinación. Ve un vínculo directo entre esa estructura y la forma en la que han progresado las tecnologías reproductivas. Considera que las relaciones de dominio son parte nuclear de la tecnología de reproducción e insiste en la importancia de pensar en la dimensión política de la tecnología. No se trata de dar continuidad a la discusión simplista de si son tecnologías buenas o malas sino que "es preciso criticar tanto los principios más básicos de estas tecnologías como sus métodos" (Mies y Shiva, 2014: 291). Y esos principios se edifican, precisamente, en la explotación de las mujeres, al mismo tiempo que los métodos se basan en el férreo control de los cuerpos femeninos y en la vigilancia médica androcentrada. Cuestiona los discursos por los que la tecnología reproductiva ha intentado obtener compasión social y aceptación. En especial, critica el relato en torno al deseo de la maternidad y la paternidad. Mientras se desarrollan los argumentos interesados para favorecer a la industria reproductiva y a la narrativa del deseo de crear familias genéticamente descendientes, no sucede, sin embargo, que haya una firme defensa en garantizar los derechos reproductivos de las mujeres.

Mientras tanto, en los últimos años, hemos presenciado un cambio considerable en el incremento del diagnóstico de esterilidad, siendo muy llamativo que se haya producido al tiempo que han ido floreciendo los tratamientos artificiales auspiciados por la industria reproductiva (Harrison, 2016). González y Pérez (2002: 107) destacan la importancia de "ocuparse críticamente de las tecnologías, prácticas y discursos que definen los cuerpos de las mujeres como deficientes" y señala con asombro el rápido giro que ha recibido la definición de esterilidad, la cual ha sido reducida a la dificultad de conseguir un embarazo en un tiempo determinado, así como su interesada identificación con la enfermedad. Mientras que a la dificultad de embarazarse no se le aplican terapias realmente resolutivas, la industria reproductiva impulsa, promueve y publicita la creación de maternidades tecnológicas.

\section{LAS REFERENCIAS EMPÍRICAS SOBRE LA GESTACIÓN SUBROGADA}

Los servicios de gestación subrogada se han distribuido extensamente en el mapa internacional. Aunque podríamos decir que Estados Unidos es el hub de esta industria, países como la India, Tailandia, México, Ucrania, Kenia y Nigeria han destacado en las dos últimas décadas por contar con clínicas especializadas. Debido a la concentración en la India, gran parte de los estudios empíricos han tenido lugar en las clínicas y servicios derivados de dicho país. Es 
por ello, que los estudios etnográficos más referenciados en la literatura académica centran sus análisis en la situación de los embarazos comerciales de la India. En general, en dichos estudios (ver Pande, 2014; Rudrappa, 2015; Twine, 2015; Harrison, 2016), se aprecia un discurso común por parte de las clínicas, las agencias implicadas y las familias, en el que se defiende la práctica como un medio para combatir el empobrecimiento de las mujeres. Se define la subrogación como un acuerdo entre iguales y sin embargo, todas las evidencias apuntan al origen y consecuencias desiguales de esta práctica (Krolokke y Pant, 2012, citando a DasGupta \& Das Dasgupta, 2010). Las parejas contratantes proceden de contextos sociales medio altos y altos, mientras que las mujeres que gestan tienen un estatus social bajo y muy bajo. De hecho, uno de los motivos que hizo proliferar exponencialmente el número de clínicas y agencias en la India fue el coste llamativamente inferior de la gestación (Schurr, 2018). Una causa común entre las mujeres que gestan para otros es el deseo de percibir ingresos que les permitirán construir una casa y pagar la educación de sus hijos e hijas. Es decir, aquello que las impulsa es la necesidad de cubrir algunos de los derechos fundamentales. Habitualmente, expresan que logran cumplir ambos deseos, gastando todo el dinero percibido y quedando de nuevo en una situación de pobreza estructural y sin perspectivas de empleo.

Aunque es difícil establecer el inicio de todo el circuito que envuelve el conglomerado de servicios que operan en este mercado internacional, tal vez sea interesante comenzar por cómo se produce la captación de las mujeres que más tarde accederán a ser vientres de alquiler. La mayor parte de agentes de captación que las atraen a las clínicas son personas de sus familias políticas. En muchos casos, son mujeres captadoras con las que se tiene cierto grado de confianza y respeto. Es habitual que sean los maridos, o parejas masculinas de las mujeres gestantes, quienes se encarguen de percibir las retribuciones. Reciben los pagos y los gestionan bajo su criterio. En el estudio de Amrita Pande (2014), algunos de estos varones son quienes firman los contratos y reparten los ingresos entre su familia extensa. Casi la mitad de las entrevistadas habían llegado a la subrogación mediante la coerción ejercida por la familia del marido y no tenían control sobre las retribuciones del proceso de gestación comercial en el que estaban inmersas.

Pande (2014) recoge numerosos testimonios de mujeres involucradas en la industria reproductiva como vientres de alquiler. Describe en profundidad todo aquello que implica el embarazo mercantilizado, como el control médico diario y el confinamiento de las mujeres contratadas en las instalaciones de las clínicas reproductivas. De las historias de vida que contiene el estudio, cabe destacar el testimonio en el que Rita, antes de gestar comercialmente dos criaturas, había vendido sus óvulos por un total de diez veces. Alude al dolor intenso que ha atravesado durante todos los procedimientos y comenta sus sensaciones de cansancio, así como las preocupaciones por alimentar a su familia, en un contexto en el que India tiene el mayor número de muertes por maternidad y sólo 51 personas médicas cada 100.000 habitantes (Pande, 2014). Pese a ello, existe, dentro del país, una fortísima inversión en nuevas tecnologías reproductivas para la gestación subrogada. Atendiendo a la investigación, una de las mujeres candidatas fue descartada como ovo- 'donante', pues fue considerada 'demasiado' mayor. Sin embargo, se le ofreció ser vientre de alquiler y, en el proceso de medicalización, se supo que estaba embarazada de forma natural de su propia criatura. La clínica le propuso el aborto, de tal manera que pudiera estar disponible para gestar el embrión de laboratorio. Decidió abortar y comenzar un proceso de gestación comercial, para poder obtener ingresos y abastecer su subsistencia, junto con la de su familia. La criatura ajena tenía un precio de mercado de varios miles de dólares.

Algunas parejas entrevistadas por Pande (2014) revelan una posición de vigilancia sobre los fines de la mujer gestante. Emiten juicios y valoran positivamente a las mujeres si el gasto de los ingresos se hace sobre las necesidades básicas de la familia, mientras que critican la compra de un televisor. También valoran la abnegación y la entrega plena de las mujeres hacia sus familias.

En una investigación de características similares, Sharmila Rudrappa (2015) habla del control de la fertilidad y de los procesos de esterilización llevados a cabo en las mujeres en décadas anteriores en la India. La mayoría de mujeres en situación de gestación comercial entrevistadas habían sido esterilizadas permanentemente. Lo que no les impedía someterse a un proceso de intensa hormonación, para después serles implantados los embriones fecundados en laboratorio. Este perfil de mujer es muy demandado por las clínicas, porque pueden reforzar en las familias contratantes la idea de que no habrá ninguna posibilidad de interferencia entre la madre gestante y la madre genética. Aunque tienen capacidades para dar a luz vaginalmente, la mayor parte de ellas son obligadas a pasar por una cesárea programada. Esto es una preferencia de la clínica y de la demanda, pues se aseguran la fecha del nacimiento y pueden concretar calendarios con las familias, que viajan desde países del Norte Global. Al mismo tiempo, les permite descartar las posibles complicaciones de un parto natural, lo que reduce el riesgo y garantiza la posterior entrega de la criatura encargada. Un dato destacado del estudio, es que las mujeres no reciben habitualmente cuidados tras la cesárea. Sólo en el caso de que ellas mismas quieran y puedan financiarlo o sean las familias contratantes quienes se lo ofrezcan. Las clínicas se ocupan de la mujer gestante hasta que entrega la criatura-producto y la salud de ellas sólo es relevante en tanto cuerpos que gestan, lo que está en el marco de un trato deshumanizado y deshumanizador.

En síntesis, vemos que la gestación subrogada es una práctica alejada de la empatía y de los derechos reproductivos de las mujeres. La gestación para otros requiere una intensa medicalización, un fuerte control clínico y un confinamiento de las mujeres durante todo el proceso. Desea a las mujeres gestantes en tanto instrumentos de gestación. Pese a poner la vida misma para obtener unos ingresos que serán empleados en conseguir los derechos básicos de educación y habitabilidad, las cantidades que perciben por atravesar un proceso de dolor frecuente, no conllevan una salida de la vulnerabilidad social y de la precariedad laboral.

\section{¿TRABAJO GESTACIONAL?}

Es difícil hablar de trabajo cuando nos referimos a la gestación subrogada, pues estamos abordando un sistema 
de explotación que tiene que ver con la creación de vida humana. Afirma Alicia Puleo (2017) que con las tecnologías reproductivas las mujeres son consideradas una materia prima. La industria reproductiva establece unos requisitos a las mujeres para ser contratadas y todos ellos están asociados al 'ser' y no al 'hacer'. Por ejemplo, las mujeres no deben tener más de cuarenta años, tienen que contar con al menos una criatura parida, no deben estar amamantando, se les pide no haber tenido más de tres embarazos, así como ningún aborto en su historial y es imprescindible contar con un útero calificado como saludable. Es decir, los requisitos se relacionan con ciertas características biológicas, no con aquello que una mujer puede hacer, aprender, mejorar, prolongar, desarrollar o profesionalizar mediante sus habilidades y destrezas. Por lo tanto, el análisis fundamental debe realizarse desde el 'ser' (igual a proceso vital), no desde el 'hacer' (igual a trabajo). Esto nos conduce a la primera laguna de la propuesta del 'trabajo gestacional' ¿un proceso vital puede ser un trabajo, aunque esté plenamente afectado por la tecnología?

Se han publicado algunas propuestas académicas que defienden el 'trabajo gestacional' como marco de interpretación de esta práctica (ver Boris y Salazar, 2010; Cooper y Waldby, 2014; Lewis, 2017, 2018, 2019). Por ello, en este apartado deseo reflexionar críticamente sobre los argumentos que defiende Sophie Lewis (2017). Opina que la gestación subrogada abre posibilidades para la fluidez de género y la proliferación de los roles de género. Asocia el embarazo natural con un discurso esencialista de la maternidad que ella denomina cisgenerizada. La cuestión de la identidad y de la multiplicidad de géneros es el núcleo de su análisis, obviando al sujeto político mujer. Considera que hablar de gestación natural es una alianza con la normatividad patriarcal. Es decir, analiza el embarazo como un constructo cultural y plantea que hacer una distinción entre la gestación subrogada y la reproducción natural conduce a una dicotomía confusa. Sin embargo, la capacidad de gestar de las mujeres no forma parte de un rol de género o de un proceso de socialización que emane de la cultura patriarcal. La capacidad de embarazarse no es una estructura política impuesta ni un comportamiento desarrollado por la opresión de género. Tampoco dicha capacidad deviene de la construcción de una identidad de género.

Con todo, Lewis (2017) insiste en rebatir el significado de la gestación natural y opina que no se pueden considerar las categorías 'mujer', 'ser humano' y 'máquina' como inmutables. Dado que opina que distinguir entre máquinas y personas es un pensamiento con un fundamento transfóbico, propone formular otras posibilidades: a) que el embarazo sea una máquina, no una mujer; b) que una persona embarazada no es necesariamente una mujer y c) que una máquina puede ser parte de una mujer. Sobre esta última idea defiende que "es urgente re-evaluar a la subrogada² gestacional comercial como una reproductora cyborg" (Lewis, 2019: 121). También

\footnotetext{
2 El texto de Sophie Lewis (2017) no contempla una complejidad en el género de las palabras porque el uso del inglés permite cierta neutralidad. Por ese motivo, he traducido sus expresiones en femenino. Pero, dado el contenido de su discurso y su posicionamiento, me inclinaría a considerar que la traducción de sus términos podría querer ser a la/al/a le subrogadx, pues son estrategias habituales que se emplean en el discurso trans y queer.
}

tilda de transfóbico al feminismo que se posiciona en contra de la gestación subrogada. Como diría Rosa María Rodríguez (2019), la deriva trans insiste en culpabilizar a las mujeres por lo que sus cuerpos poseen de biológico. Y como expresaría Valcárcel (2019), el sufijo -fóbico se aplica desde esta deriva a toda voz disidente de sus posiciones, como estrategia para mitigar el debate feminista. Pero ya hemos comprobado que la industria de los vientres de alquiler impone requisitos biológicos a las mujeres que van a gestar para otros ¿cómo hablar, pues, de máquinas que gestan? ¿no sería, en cualquier caso, el sistema de gestación subrogada quien mantiene una posición transfóbica en su seno? Aun así, Lewis (2017) insiste en plantear sus formulaciones para combatir una supuesta transfobia y tecnofobia en las posiciones abolicionistas de dicha práctica.

Lewis (2017) se opone al lema "A woman is a human being, not a machine" ("una mujer es un ser humano, no una máquina") del colectivo feminista Stop Surrogacy Now. Según Mercedes Expósito (2004), las mujeres quedan determinadas por la técnica, una práctica que parte de la idea de dominación y explotación de la naturaleza, siempre asociada al concepto de mujer. "Para la tecnología, el organismo es una máquina" (Expósito, 2004: 188) y así se manifiesta, no sólo en el discurso teórico de Lewis (2017), sino en la nueva segregación de la maternidad en distintas piezas que se unen como en una cadena de ensamblaje. En la gestación comercial, además de intervenir diversos agentes involucrados en el negocio (farmacéuticas, servicios jurídicos, agencias de viaje, agencias de subrogación, clínicas reproductivas, entre otros), son parte fundamental el material genético (óvulos, esperma), que pueden proceder de la pareja interesada o de bancos de donación. Se ensamblan la creación de los embriones en el laboratorio, su implantación en el útero de la mujer subrogada, el proceso de gestación, la cesárea programada y la entrega del o de la bebé a las personas que hayan contratado el servicio.

Lewis (2017), aunque reconoce que la estructura de los mercados de gestación comercial es neocolonial, considera que dicha aglomeración empresarial trasnacional puede padecer los efectos del 'estigma' de la gestación subrogada. Ve en el uso de la expresión 'mujeres utilizadas en los sistemas de subrogación' una negación de la agencia de éstas. Son ambas ideas importadas del discurso pro regulación de la prostitución, por lo que propone que las partes interesadas en el negocio aprendan de los métodos empleados por el movimiento a favor de la descriminalización del 'trabajo sexual' - es decir, la regulación del proxenetismo, ya sea sexual o reproductivo-, de forma que pueda facilitarse la legitimación social y jurídica de esta actividad. Así, es preciso aclarar que señalar y visibilizar el hecho de que las mujeres son utilizadas por una industria que emplea la desigualdad de género como forma original de negocio, no es desdeñar la agencia de éstas, sino examinar la dimensión política de la práctica. No podemos olvidar que, en la gestación para otros en numerosas clínicas de la India, se establece la obligatoriedad de permanecer en los dormitorios durante todo el proceso, lo que dota a la industria de la capacidad de controlar plenamente la vida de las mujeres durante el tratamiento de hormonación, el embarazo y el parto. No tienen libertad deambulatoria, no se les permite hacer ninguna otra actividad externa al dormitorio, no pueden 
mantener relaciones sexuales ni cuidar de sus propias familias. En estas circunstancias no se produce un ejercicio de los derechos esenciales, ¿'estamos pues en el plano de la agencia individual o en el de la violencia estructural de género?.

\section{LA MARGINACIÓN DEL SUJETO POLÍTICO MUJER}

Lewis (2017: 115) opina que la declaración de que una mujer es un ser humano y no una máquina, tiene encriptado el mensaje: "una gestante debe ser lo que nosotras [las feministas anti vientres de alquiler] entendemos que es una mujer, la cual definimos en oposición a la artificial" y cree que "la preocupación por diferenciar el sujeto que gesta de la máquina" responde a la "insistencia de la esencia natural de la maternidad cisgenerizada". Aquí, Lewis (2017) ha añadido una complejidad que nace de la teoría de la identidad. El embarazo en una mujer no surge de una idea insistente, es un proceso biológico, real y evidenciado, no es un proceso cultural. La defensa de que una mujer es un ser humano en el contexto de la gestación comercial obedece al reconocimiento necesario de los derechos básicos de las mujeres. La teoría feminista ya se encargó de señalar que es la cultura patriarcal quien define todos aquellos significados que se encuentran en oposición a la mujer. Gran parte de ellos son los que construyen seres plenamente humanos, que emanan de la socialización jerárquica de géneros y que se atribuyen universalmente a los varones. Por otro lado, la preocupación de las feministas por seguir recordando que las mujeres deben ser reconocidas como seres humanos, no obedece a ninguna postura esencialista, en todo caso, defiende una realidad incuestionable, es decir, que es preciso seguir señalando que los derechos reproductivos forman parte de los derechos humanos y que por ello, el feminismo vindica la esencia humana de las mujeres.

Más parece, sin embargo, que la duda de qué es una mujer es una preocupación que obsesiona a Lewis (2017), aspecto que puede saldar acudiendo al pensamiento feminista. Este entuerto intencionado de tener que explicar a qué nos referimos cuando mencionamos la palabra mujer, ha sido una suspicacia que ha traído el relato de las identidades. El feminismo, desde su fundamento ilustrado, ha venido definiendo con claridad todos los significados políticos, sociales, culturales y económicos de ser mujer en las sociedades patriarcales. Lo que hace Lewis (2017) al marginar la atención al sujeto político mujer, es evidenciar que su agenda pone en el centro el juego de los géneros y de las identidades frente a las complejidades, retos y servidumbres, en definitiva, frente a la violencia política que sobre las mujeres pone la gestación para otros.

Mientras tanto, parece que la propuesta de Lewis (2017: 121) "el embarazo es una máquina, no una mujer" se materializa progresivamente. En octubre de 2019 el canal BBC presentaba la noticia sobre la inminente llegada del útero artificial para seres humanos. Un equipo de investigación de la Universidad Tecnológica de Eindhoven (Holanda) celebraba la concesión de 2.9 millones de euros para el desarrollo y el perfeccionamiento de su proyecto. El artefacto, ideado, en principio, para incubar a bebés que nacen muy prematuramente, se plantea también como una posible manera de gestar artificialmente, una forma de externalizar el embarazo. Así se expresaba la creadora del prototipo, Lisa Mandemaker:

"Imagino que en un futuro el útero artificial podría llegar a ser parte de un estilo de vida para las mujeres, porque no han de preocuparse por el malestar matutino, los cambios en su cuerpo...creo que podría ser muy interesante para algunas personas, por ejemplo, podemos pensar en los hombres gays. Me parece que hay en la sociedad un relato de que existe una forma ideal de reproducción natural. La reproducción natural no es la única forma" ("The world's first artificial womb for humans", 2019).

Como vemos, la creadora también defiende la intervención tecnológica extrema en la gestación de un ser humano e incita a un pensamiento confuso sobre la maternidad. Menciona que las sociedades 'creen' que la vida 'solo' se gesta de forma natural en una mujer y proyecta un futuro en el que un útero artificial puede convertirse en una elección para formar una familia. Pero sin duda alguna, la gestación natural no es una creencia, es un hecho empírico, probado, que todo ser humano ha nacido de una mujer a lo largo de la existencia de la humanidad.

Si acudimos al legado de Simone de Beauvoir (1999), quien realizó un esfuerzo por explicar la construcción de lo humano en las sociedades patriarcales, comprendemos que las mujeres son diferenciadas principalmente de los hombres por ocupar un segundo lugar, el espacio simbólico y material subsidiario. En la medida en que lo humano se ha construido con aquello que es semejante al hombre y a lo masculino, la mujer es la idea opuesta. Enfrentada, deficiente y devaluada con respecto al modelo universal androcéntrico de humanidad, la mujer no ha llegado a alcanzar la consideración completa perteneciente al ser humano y por eso es, el segundo sexo. Por otro lado, la antítesis de lo humano es también la máquina, creada para producir y reproducir de forma ilimitada. Las máquinas carecen de empatía, de sentimientos y de emociones, es decir, las máquinas carecen de elementos humanos. Sin embargo, todos los procesos vitales en los seres humanos contemplan emociones. Los embarazos son procesos vitales, no son máquinas. Dado que Lewis (2017) utiliza a las mujeres desde un imaginario que las aproxima a las máquinas, considero que es una acepción que las conduce a ser consideradas seres menos humanos, no-humanos, o más concretamente, deshumanizados. Se constituye con su propuesta una y otra vez el segundo sexo. En la medida en que las mujeres están próximas a las máquinas, se las conceptualiza también como obedientes, disponibles, manejables, complacientes, abnegadas. Entramos entonces en el terreno en el que pueden ser programadas de acuerdo a los deseos de otros. Son las arenas movedizas de vivir para otros y, sobre todo, de gestar para otros. Estos planteamientos refuerzan el rol de la feminidad y no contribuyen a la emancipación colectiva de las mujeres.

\section{LA 'GESTANTE PROFESIONAL': BIOCAPITALISMO Y SEXISMO MISÓGINO}

Lewis (2017) sostiene que gestar para otros ha de entrar dentro de la consideración feminista del trabajo reproductivo. Sabemos que el trabajo reproductivo, como ha instado la economía feminista y las feministas 
marxistas, es el reconocimiento de todas las acciones y servicios imprescindibles para el sostenimiento de la vida, que tradicionalmente han ejercido -y ejercen- las mujeres en prácticamente todas las sociedades del mundo. Pero la gestación subrogada no es verdaderamente imprescindible para el sostenimiento de la vida. No podemos ubicar la gestación para otros en el marco de los costes sociales que recaen en las espaldas de las mujeres para que el sistema productivo prospere. Es un nicho de mercado que la industria tecnológica y reproductiva ha encontrado en la fase de capitalismo actual. Las feministas y ecofeministas mantienen su oposición a esta práctica porque se mercantiliza un proceso vital. Es la entrada del embarazo en la lógica capitalista. La gestación subrogada no cumple con los postulados de la economía feminista, que aboga por poner la economía al servicio de la vida. Todo lo contrario, conlleva poner la vida al servicio de la economía, es una lógica biocapitalista, es "una extensión del mercado dentro de la esfera de la reproducción" (Balaguer, 2017: 180).

Lewis (2017) persigue construir un discurso que dé permisividad social y permeabilidad legal a los vientres de alquiler. Inventa una serie de neologismos que borran las palabras 'mujer embarazada' y las substituye por commercial gestational surrogate, pregnancy worker, surrogate gestator, surrogate, professional surrogate, hired gestator, subject of gestation ${ }^{3}$. Estos conceptos comportan, por un lado, incoherencias con la realidad y, por otro, algunos entorpecimientos en el progreso de la articulación social y política del feminismo en las sociedades. Aunque expone que la postura académica y activista anti subrogación no ha trascendido el nivel de la abstracción, el análisis de su lenguaje me permite recorrer el terreno de lo concreto. Sobre la expresión professional surrogate (subrogada profesional), cabría preguntarse: ¿qué es un embarazo 'profesional'? Las mujeres que acceden a ser vientres de alquiler no han tenido una formación profesional que las haya cualificado para desarrollar embarazos 'profesionales', por lo tanto, no es una denominación apropiada. No reciben ningún tipo de diploma profesional, ni supone una inversión de tiempo que pueda tener una continuidad. El contrato de gestación subrogada no es una mejora en sus posibilidades futuras de empleo, no comporta una transformación en sus vidas laborales a largo plazo, menos aún una solución estructural, por lo tanto ¿conlleva la gestación comercial un posible apartado para el curriculum de las mujeres como experiencia profesional en el mundo actual? ¿qué capacidades desarrollan en esa práctica que les puedan ser de utilidad para conseguir un empleo y unos ingresos continuados a posteriori?

Ser vientre de alquiler es una ocupación temporal y puntual en sus vidas, no es una ocupación que solucione la falta de ingresos, la precariedad y la inestabilidad de recursos para la supervivencia. Además, surgen las cuestiones: si el embarazo en la gestación subrogada es considerado un trabajo como cualquier otro y está en el rango de lo profesional ¿cómo afecta esa idea a la baja por maternidad? ¿pueden las mujeres que gestan para otros acceder a una baja por maternidad? ¿si es 'trabajo gestacional' es también

\footnotetext{
3 Traduciríamos por: subrogada gestacional comercial, trabajadora del embarazo, gestante subrogada, subrogada, subrogada profesional, gestante contratada, sujeto de gestación.
}

maternidad?

La conceptualización de la gestación para otros como 'trabajo gestacional' a menudo recae en la idea de que las mujeres implicadas en el embarazo sólo aportan su útero. Sin embargo, el útero es indisociable de la mujer que gesta, es decir, esta práctica conlleva la presencia constante y continuada de la mujer en su totalidad y a tiempo completo. No se produce ningún descanso de la mujer, pues toda ella está ofrecida sólo para la gestación y controlada por la clínica. Es una situación más próxima a la esclavitud que al empleo.

En una interesante contribución teórica crítica con el marco de interpretación del 'trabajo gestacional' Johanna Oksala (2019) señala que Melinda Cooper y Catherine Waldby, autoras pro subrogación destacadas, admiten que esta visión es la entrada del capitalismo en la dimensión de la vida misma. Es decir, el biocapitalismo explota algo que va mucho más allá de la plusvalía obtenida por la mano de obra de las personas. Por ello, defienden que la solución a esta complejidad sería que las mujeres que gestan para otros fueran tenidas en cuenta como propietarias de un recurso natural (su útero), el cual deben poner en valor. Vendría siendo algo así como la consideración mercantil de la capacidad reproductiva incorporada en la lógica de la propiedad privada y funcionando a modo de 'capital reproductivo'. Pero este paradigma comporta algunos problemas. Explica Oksala (2019) que dichas mujeres no pueden realmente actuar como propietarias de su recurso biológico, porque en cuanto firman el contrato de gestación subrogada, sus cuerpos pasan a pertenecer a la autoridad médica y a la familia contratante. Ellas quedan sin soberanía para decidir qué pasará con sus cuerpos y sus vidas durante toda la duración del proceso. Lo que venden las mujeres sujetas a situación de vientres de alquiler es, a todos los efectos, la pérdida total del control de sus cuerpos y de su salud (Oksala, 2019). Sobre ello, Luisa Muraro (2017: 41) también opina que "se trata de subordinar la fecundidad personal a un proyecto de otros, que serán los titulares de su fruto y dictan las condiciones de su desarrollo".

Por otro lado, siguiendo a Oksala (2019), no es posible hablar de 'trabajo gestacional' porque claramente, las mujeres que gestan comercialmente no reciben una remuneración por los servicios prestados, sino por la entrega de la criatura encargada. Esto es, si se produce un aborto inesperado o no fructifica el embarazo, las mujeres no reciben la compensación completa, sólo una mínima parte. Pueden darse otras situaciones, en las que la pareja que encargó la gestación subrogada se niegue a aceptar una criatura que haya nacido con cualquier malformación. $\mathrm{O}$, como de hecho se ha producido, uno de los casos más conocidos es el del bebé Gammy, que con síndrome de Down, fue abandonado por la pareja australiana que había comprado el embarazo comercial en Tailandia. Como argumenta Oksala (2019), a diferencia de las amas de cría y de las cuidadoras de menores, a las cuales se les retribuye el tiempo de trabajo de crianza, a las mujeres subrogadas en la industria reproductiva se les compensa por la criatura entregada tras el parto, no por su tiempo ni sus habilidades.

Denominar a las mujeres que proceden a gestar para otros 'gestante profesional', es una práctica de sexismo misógino. El sexismo se muestra cuando existe una actitud de minusvaloración hacia las mujeres, por el mero hecho de serlo. Genera un valor cultural de menosprecio hacia 
todo aquello que las mujeres son, hacen o pueden hacer y en muchos casos, se manifiesta en su invisibilización, tanto en el lenguaje, como en el trato o en el reconocimiento. La misoginia es una conducta de rechazo, de odio y aversión contra las mujeres, también motivada por el mero hecho de serlo. Una práctica de sexismo misógino es esta maniobra explícita de ocultación en el lenguaje de una realidad tan obvia como que el embarazo es un proceso biológico que sucede en las mujeres. Este sexismo misógino es el motor que lleva a la invención del listado de neologismos de Lewis (2017). Como diría Elena Simón (2008), la ocultación de las mujeres en el lenguaje consiste en no nombrarlas bajo todo tipo de subterfugios y suele dar lugar a situaciones de ambigüedad. La teoría feminista ha insistido en que aquello que no se nombra, no existe (Balaguer, 2017). Invisibilizar quiénes son las que se embarazan en la gestación subrogada y fuera de ella sólo puede conducir al menosprecio del papel fundamental de las mujeres en esta práctica. Esta ocultación no es sólo violencia discursiva. ¿Cómo van a ser atendidas las necesidades específicas de las mujeres que están en situaciones de vientres de alquiler si no son nombradas y las hacen desaparecer, como propone Lewis (2017)?

La cuestión sobre las necesidades específicas no es baladí. Las mujeres que gestan en esta modalidad se someten a un tratamiento muy similar a las mujeres que venden sus óvulos. Atendiendo a los riesgos sobre la salud de las mujeres, recogidos por Octavio Salazar (2018), es preciso mencionar, por ejemplo, los cánceres reproductivos, los infartos cerebrales y, en algunos casos, la muerte. Todavía miles de mujeres fallecen por falta de los medios adecuados para la atención al parto y al postparto (Valcárcel, 2019), así como por no disponer de los medios y legislaciones oportunas para el aborto libre, gratuito y seguro. Vivimos en sociedades en las que es aun dificultoso optar a un empleo o conservarlo si las mujeres son susceptibles de, o están, en proceso de gestación. Son numerosas las circunstancias específicas de discriminación que las mujeres sufren en el mundo relacionadas con el embarazo en las sociedades patriarcales y capitalistas, en un contexto social y cultural que todavía insiste en invisibilizarlas mediante el lenguaje. Ocultar la participación ineludible de las mujeres en los embarazos, sean comerciales o no, supone un proceso violento de manipulación de la realidad de éstas, que da lugar a un sexismo misógino: depreciación, devaluación, ocultación y una brizna de odio. La supresión interesada conlleva que las necesidades concretas de las mujeres durante la gestación, puedan no tenerse en cuenta. La complejidad de esta situación se agrava por la enorme medicalización de las mujeres en la gestación para otros, así como en todo el contexto de experimentación médica que realiza la industria reproductiva con los cuerpos femeninos, cuyos impactos todavía se desconocen en profundidad (Pérez y Ortega, 2014).

Se trata de un sexismo misógino de unos impactos extensos, en lo plenamente estructural. Así, la gestación subrogada se desarrolla en nuestras sociedades patriarcales con un abanico de retos en su génesis y expansión, que en síntesis es para las mujeres un sistema de riesgos para su salud, cosificación extrema de sus cuerpos, pervivencia de las servidumbres femeninas en sus dimensiones sociales y económicas y violencia política contra ellas. Pero, pese a estos elementos intrínsecos a esta práctica, Lewis (2017) considera que el problema sobre el que orbitan las desventajas de la gestación comercial es su falta de regulación. Propone crear un discurso en torno a estos planteamientos, con el fin de activar el camino hacia la completa legalización de los vientres de alquiler:

"los espacios de trabajo de la subrogación son en última instancia irremediablemente peligrosos porque...los efectos de la subrogación no regulada en las vidas de las personas son abrumadoramente violentos en las siguientes maneras...la subrogación debe ser descriminalizada (con el fin de hacerla más segura) porque..." (Lewis, 2017: 115).

Por lo cual, desde un punto de vista centrado en los problemas intrínsecos de la práctica y que se originan en la estructura patriarcal y en la explotación de los cuerpos de las mujeres, cabría dar un giro opuesto al discurso, de manera que nos permita dar pie a investigaciones que persigan desactivar todas las violencias de su sistema y habilitar los caminos para la abolición de la gestación subrogada. Los planteamientos serían modificados por: los espacios de explotación estructural de la subrogación son irremediablemente peligrosos porque...la subrogación es intrínsecamente una práctica abrumadoramente violenta contra las mujeres en las siguientes maneras...la subrogación debe ser abolida (con el fin de progresar en la articulación de sociedades feministas) porque....

\section{CONCLUSIONES}

A lo largo de la reflexión aquí presentada se ha comprobado que la gestación subrogada tiene importantes implicaciones específicas en la situación de las mujeres, sobre las cuales, todavía se pone en tela de juicio su valor humano. En el discurso de Sophie Lewis (2017) no hay realmente una preocupación por lo que sucede con las mujeres en la gestación subrogada. Su prioridad es la preocupación por la fluidez de género. Se evidencia con ello un fuerte desequilibrio en sus preocupaciones desde el punto de vista de la teoría feminista. Que afirmar el valor humano intrínseco de las mujeres sea leído por Lewis (2017) como una postura transfóbica y tecnofóbica es un argumento que no se sostiene, pues queda constatado que parece más una estrategia discursiva para desacreditar los planteamientos abolicionistas, que una idea fundamentada en el pensamiento feminista. Se comprueba que las propuestas de Lewis (2017) tienen características clásicas de las prácticas androcéntricas, con una intención insistente por deshumanizar e interponerse tecnológicamente en el proceso vital que es la gestación. Su deriva es combustible para intervenir, maquinizar y artificializar sin límites el embarazo. Es la experimentación androcéntrica de las mujeres llevada al extremo. Es precisamente por la dimensión y la especificidad de estas implicaciones, que no podemos realizar una evaluación del fenómeno si eliminamos a las mujeres del discurso.

La orientación y el juego de identidades de género que plantea Sophie Lewis (2017) desprende un conjunto de discriminaciones hacia las mujeres que dificultan el avance de la agenda feminista sobre los derechos reproductivos, así como sobre el reconocimiento, la representación y el trato hacia éstas. El discurso examinado sostiene un sesgo evidente sobre la realidad de quiénes gestan en el mundo y 
en los vientres de alquiler. Olvida que la industrialización de la gestación contribuye, como pieza clave, a generar cadenas trasnacionales de explotación reproductiva femenina. Este sesgo es contemplado por su parte, como una opción de avance hacia la fluidez de género, pero se desprende que su apuesta por hacer del embarazo una práctica de máxima tecnificación e interpretarlo como 'trabajo gestacional' ofrece como resultado un sexismo misógino y un uso instrumental de los cuerpos de las mujeres, que lleva a su deshumanización y cosificación. Al mismo tiempo, proletarizar y maquinizar la maternidad significa que se margina la experiencia humana para orientarse hacia el paradigma biocapitalista y ello conduce directamente a la explotación reproductiva extrema de las mujeres. Así pues, la gestación de la vida en esta práctica se ejerce desde una idea basada en el mercado. Lo que conlleva, al mismo tiempo, una aportación más a la conceptualización de las mujeres como seres de consumo y para el consumo de otros. No es sólo una cuestión de biodisponibilidad, sino la disponibilidad absoluta de la vida misma. Por ello, queda totalmente descartada la maternidad cyborg como una opción feminista viable.

El control de las mujeres en la gestación subrogada adquiere su máxima expresión en los espacios-dormitorio, donde la disponibilidad a tiempo completo, la entrega de la soberanía de sus cuerpos a la autoridad médica y el confinamiento son, en esencia, equivalentes a la esclavitud. Se constata que el uso de las tecnologías reproductivas en esta práctica se realiza con una dimensión política, es decir, desde el poder y la desigualdad, por lo que es preciso no olvidar que la investigación feminista sobre los vientres de alquiler ha de ser política. Dado el escenario de violencias específicas contra las mujeres que plantea la gestación subrogada y los discursos académicos que pretenden impulsar su regulación jurídica y su legitimidad social, considero necesario seguir elaborando un marco de interpretación abolicionista, que permita profundizar en los múltiples impactos y complejidades que van apareciendo en su desarrollo. Todo ello hará posible la ampliación de propuestas de desarticulación de la explotación reproductiva de las mujeres y seguir trazando caminos para la articulación de sociedades feministas en las que las mujeres puedan ser sujetos de pleno derecho en su humanidad completa.

\section{REFERENCIAS}

Balaguer, Maria Luisa. 2017. Hij@s del mercado. La maternidad subrogada en un Estado Social. Madrid: Cátedra.

"The world's first artificial womb for humans". 2019. BBC. 16/10/2019 https://www.bbc.com/news/av/ health-50056405/the-world-s-first-artificial-womb-forhumans

Beauvoir, Simone de. 1999. El segundo sexo. Madrid: Cátedra. Boris, Eileen y Salazar, Rhacel (eds). 2010. Intimate Labors: Cultures, Technologies, and the Politics of Care. Stanford CA: Stanford University Press.

CCMA. 2016. Nens que no vénen de París. España: CCMA. https://www.ccma.cat/tv3/alacarta/30-minuts/nensque-no-venen-de-paris/video/5557406/

Cooper, Melinda y Waldby, Catherine. 2014. Clinical Labor: Tissue Donors and Research Subjects in the Global
Bioeconomy. Durham NC: Duke University Press.

Corea, Gena. 1988. The mother machine: reproductive technologies from artificial insemination to artificial wombs. London: Women's Press.

Expósito, Mercedes. 2004. La maternidad en el siglo XXI: una construcción imaginario-tecnológica. Thémata, Revista de Filosofía, 33: 185-90.

González, Marta, y Pérez, Eulalia. (2002). Ciencia, tecnología y género. $O E I$, Enero-Abril.

Harrison, Laura. 2016. Brown Bodies, White Babies. The Politics of Cross-Racial Surrogacy. New York Press.

Kroløkke, Charlotte Halmø y Pant, Saumya. 2012. "I only need her uterus": Neo-liberal Discourses on Transnational Surrogacy. NORA - Nordic Journal of Feminist and Gender Research 20 (4): 233-48. https://doi.org/10.1080/080387 40.2012.729535.

Lewis, Sophie. 2017. Defending Intimacy against What? Limits of Antisurrogacy Feminisms. Signs: Journal of Women in Culture and Society 43 (1): 97-125. https://doi. org/10.1086/692518.

Lewis, Sophie. 2018. International Solidarity in reproductive justice: surrogacy and gender-inclusive polymaternalism. Gender, Place \& Culture 0524: 1-21. https://doi. org/10.1080/0966369X.2018.1425286.

Lewis, Sophie. 2019. Full Surrogacy Now. London and New York: Verso.

Martínez, Layla. 2019. Gestación subrogada. Capitalismo, patriarcado y poder. Logroño: Pepitas.

Mies, Maria y Shiva, Vandana. 2014. Ecofeminismo. Barcelona: Icaria Editorial.

Muraro, Luisa. 2017. El alma del cuerpo. Barcelona: Icaria Editorial.

Nuño, Laura. 2016. Una nueva cláusula del Contrato Sexual: vientres de alquiler. Isegoría 55 (diciembre): 683. https:// doi.org/10.3989/isegoria.2016.055.15.

Oksala, Johanna. 2019. Feminism against Biocapitalism: Gestational Surrogacy and the Limits of the Labor Paradigm. Signs: Journal of Women in Culture and Society 44 (4): 883-904. https://doi.org/10.1086/702031.

Pande, Amrita. 2014. Wombs in Labor: Transnational Commercial Surrogacy in India. Columbia University Press.

Pérez, Eulalia y Ortega, Esther (eds). 2014. Cartografías del cuerpo. Madrid: Cátedra.

Puleo, Alicia. 2017. Nuevasformas de desigualdad en un mundo globalizado. El alquiler de úteros como extractivismo. Revista Europea de Derechos Fundamentales 29: 165-84.

Raymond, Janice. 1989. At Issue Reproductive Technologies, Radical Feminism and Socialist Liberalism. Reproductive and Genetic Engineering: Journal of International Feminist Analysis 2 (2).

Rich, Adrienne. 2019. Nacemos de mujer. La maternidad como experiencia e institución. Madrid: Traficantes de sueños.

Rodríguez, Rosa María. 2019. La mujer molesta. Feminismos postgénero y transidentidad sexual. Editorial Ménades.

Rudrappa, Sharmila. 2015. Discounted Life. The Price of Global Surrogacy in India. New York: NYU Press.

Salazar, Octavio. 2018. La gestación para otros. Madrid: Dykinson.

Schurr, Carolin. 2018. The baby business booms: Economic 
geographies of assisted reproduction. Geography Compass 12 (8): 1-15. https://doi.org/10.1111/gec3.12395.

Simón, Elena. 2008. Hijas de la igualdad, herederas de injusticias. Madrid: Narcea.

Twine, France Winddance. 2015. Outsourcing the womb. Race, class and gestational surrogacy in a global market. Routledge.

Valcárcel, Amelia. 2019. Ahora, feminismo. Cuestiones candentes y frentes abiertos. Madrid: Cátedra.

Wajcman, Judy. 2006. El tecnofeminismo. Madrid: Cátedra. 